R. Takagi

Nagoya Math. J.

Vol. 91 (1983), 1-18

\title{
ON HIGHER COVARIANT DERIVATIVES OF THE CURVATURE TENSORS OF KÄHLERIAN $C$-SPACES
}

\author{
Dedicated to Professor I. Mogi on his 60th birthday
}

\section{RYOICHI TAKAGI}

A compact simply connected complex homogeneous manifold is said briefly a $C$-space, which was completely classified by H. C. Wang [12]. A $C$-space is called to be Kählerian if it admits a Kählerian metric such that a group of isometries acts transitively on it. Hermitian symmetric spaces of compact type are typical examples of a Kählerian $C$-space. Let $M$ be an arbitrary Kählerian $C$-space and $R$ its curvature tensor. $\mathrm{M}$. Itoh [6] expressed $R$ in the language of Lie algebra and investigated various properties of $R$. In this paper, we study higher covariant derivatives of $R$.

First we shall show that for each $M$ there exists a positive integer $m$ such that

$$
\underbrace{\stackrel{+}{\nabla} \cdots \stackrel{+}{V}}_{m \text { times }} R=0, \quad \underbrace{\stackrel{+}{\nabla} \cdots \stackrel{+}{V}}_{(m-1) \text { times }} R \neq 0,
$$

where $\vec{V}$ denotes the covariant derivative of $(1,0)$-type. We call the integer $m$ the degree of a Kählerian $C$-space $M$. Obviously, Hermitian symmetric spaces of compact type can be characterized as $C$-spaces with degree one.

Next we shall determine all $C$-spaces with degree two, which are stated as Theorems 4.1, 4.2 and 4.7. They will form a class of the "simplest" spaces among Kählerian $C$-spaces except for Hermitian symmetric spaces.

Our results have some applications to a theory of Kählerian submanifolds in a complex projective space. This will be discussed in a forthcoming paper [10].

\section{$\S 1$. Kählerian $C$-spaces}

In this section we recall the construction of irreducible Kählerian

Received August 25, 1981. 
$C$-spaces. For details, we refer to Borel and Hirzebruch [2], Itoh [6], Nakagawa and Takagi [8], Takeuchi [11].

Let $g$ be a complex simple Lie algebra and $\mathfrak{h}$ a Cartan subalgebra. The dual space of a complex vector space $\mathfrak{h}$ is denoted by $\mathfrak{h}^{*}$. An element $\alpha$ of $\mathfrak{h}^{*}$ is called a root of $(\mathfrak{g}, \mathfrak{h})$ if there exists a non-zero vector $E_{\alpha}$ in $g$ such that

$$
\left[H, E_{\alpha}\right]=\alpha(H) E_{\alpha} \quad \text { for } H \in \mathfrak{h} .
$$

We denote by $\Delta$ the set of all non-zero roots of $(\mathfrak{g}, \mathfrak{h})$ and put $\mathfrak{g}_{\alpha}=C E_{\alpha}$. Then we have a direct sum decomposition:

$$
\mathfrak{g}=\mathfrak{h}+\sum_{\alpha \in A} \mathfrak{g}_{\alpha}
$$

Since the Killing form $B$ of $\mathfrak{g}$ is non-degenerate on $\mathfrak{h} \times \mathfrak{h}$, for each $\xi \in \mathfrak{h}^{*}$ we can define $H_{\xi} \in \mathfrak{h}$ by

$$
B\left(H, H_{\xi}\right)=\xi(H) \quad \text { for } H \in \mathfrak{h} .
$$

The following property of $B$ is fundamental:

$$
B([X, Y], Z)+B(Y,[X, Z])=0 \quad \text { for } X, Y, Z \in \mathfrak{g} .
$$

Put $\mathfrak{h}_{o}=\sum_{\alpha \in \Delta} \boldsymbol{R} \boldsymbol{H}_{\alpha}$ and define an inner products (, ) on the dual space $\mathfrak{h}_{o}^{*}$ of a real vector space $\mathfrak{h}_{o}$ by $(\xi, \eta)=B\left(H_{\xi}, H_{\eta}\right)$. We fix a lexicographic order $<$ on $\mathfrak{h}_{0}^{*}$. Let $\Pi=\left\{\alpha_{1}, \cdots, \alpha_{\ell}\right\}$ be the fundamental root system of $\Delta$ with respect to $<$ (so $\left.\ell=\operatorname{dim}_{C} \mathfrak{h}\right)$. Put $\Delta^{+}=\{\alpha \in \Delta \mid o<\alpha\}$. For each $\alpha \in \Delta$ we select a basis $E_{\alpha}$ of $g_{\alpha}$ in such a way that $\left\{H_{\alpha_{1}}, \cdots, H_{\alpha_{\ell}}, E_{\alpha}(\alpha \in \Delta)\right\}$ forms a Weyl's canonical basis of $g$, that is, it satisfies

$$
\left\{\begin{array}{l}
B\left(E_{\alpha}, E_{-\alpha}\right)=-1, \\
{\left[E_{\alpha}, E_{\beta}\right]=N_{\alpha+\beta} E_{\alpha+\beta}, N_{\alpha+\beta}=N_{-\alpha-\beta} \in R \quad \text { for } \alpha, \beta \in \Delta .}
\end{array}\right.
$$

The first equation is equivalent to $\left[E_{\alpha}, E_{-\alpha}\right]=-H_{\alpha}$. Then the following $\mathfrak{g}_{u}$ is a compact real form of $\mathfrak{g}$ :

$$
\mathfrak{g}_{u}=\sum_{\alpha \in \Delta} R \sqrt{-1} H_{\alpha}+\sum_{\alpha \in \Delta}\left(R A_{\alpha}+R B_{\alpha}\right)
$$

where we put $A_{\alpha}=E_{\alpha}+E_{-\alpha}, B_{\alpha}=\sqrt{-1}\left(E_{\alpha}-E_{-\alpha}\right)$. The complex conjugation ${ }^{-}$on $\mathrm{g}$ with respect to $\mathfrak{g}_{u}$ is given by

$$
\bar{E}_{\alpha}=E_{-\alpha}, \bar{E}_{-\alpha}=E_{\alpha}, \bar{H}_{\alpha}=-H_{\alpha} \quad \text { for } \alpha \in \Delta^{+} .
$$

Now we choose an arbitrary non-empty subset $\Phi=\left\{\alpha_{i_{1}}, \cdots, \alpha_{i_{r}}\right\}$ of $\Pi$. Define a subset $\Delta^{+}(\Phi)$ of $\Delta^{+}$by 


$$
\begin{aligned}
\Delta^{+}(\Phi) & =\Delta^{+}\left(\alpha_{i_{1}}, \cdots, \alpha_{i_{r}}\right) \\
& =\left\{\sum_{\imath=1}^{\ell} n_{i} \alpha_{i} \in \Delta^{+} ;\left(n_{i_{1}}, \cdots, n_{i_{r}}\right) \neq 0\right\} .
\end{aligned}
$$

It is clear that if $\alpha, \beta \in \Delta^{+}(\Phi)$, then $\alpha+\beta \in \Delta^{+}(\Phi)$, and that the highest root in $\Delta$ always belongs to $\Delta^{+}(\Phi)$. Define a complex subalgebra $\mathfrak{L}_{\Phi}$ of $\mathfrak{g}$ associated with $\Phi$ by

$$
\mathfrak{R}_{\Phi}=\mathfrak{h}+\sum_{\alpha \in \Delta-\Delta+(\Phi)} \mathfrak{g}_{\alpha}
$$

If we put $\mathfrak{f}_{\phi}=\mathfrak{g}_{u} \cap \mathfrak{R}_{\Phi}$, then it is a subalgebra of $\mathfrak{g}_{u}$ expressed as

$$
\mathfrak{f}_{\Phi}=\sum_{\alpha \in \Delta} R \sqrt{-1} H_{\alpha}+\sum_{\alpha \in \Delta-\Delta+(\Phi)}\left(R A_{\alpha}+R B_{\alpha}\right) .
$$

Let $G$ be the simply connected complex Lie group with Lie algebra g. Let $L_{\Phi}$ be the connected complex Lie subgroup of $G$ with Lie algebra $\mathfrak{\Omega}_{\Phi}$, and $G_{u}, K_{\Phi}$ the connected Lie subgroup of $G$ with Lie algebras $\mathfrak{g}_{u}, \mathfrak{f}_{\Phi}$, respectively. Then we obtain an irreducible $C$-space $G_{u} / K_{\Phi}=G / L_{\Phi}$, denoted by $M(\mathfrak{g}, \Phi)$ or $M\left(\mathfrak{g}, \alpha_{i_{1}}, \cdots, \alpha_{i_{r}}\right)$. Conversely, every irreducible $C$ space can be obtained in this way ([12]).

Next we describe a $G_{u}$-invariant Kählerian metric $g$ on a $C$-space $M(\mathfrak{g}, \Phi)$. For a vector space $V$, the complexification is denoted by $V^{c}$. Put

$$
\mathfrak{m}_{\Phi}=\sum_{\alpha \in \Delta^{+}(\Phi)}\left(\mathfrak{g}_{\alpha}+\mathfrak{g}_{-\alpha}\right) \text {. }
$$

Hereafter we put $\mathfrak{m}=\mathfrak{m}_{\Phi}$ and $\mathfrak{f}=\mathfrak{l}^{C}$ for simplicity. Then we have a direct sum $\mathfrak{g}=\mathfrak{H}+\mathfrak{m}$ orthogonal with respect to $B$. Denoting the tangent space of $M(\mathfrak{g}, \Phi)$ at the origin $o=K_{\Phi}$ by $T_{o}(M)$, we can identify $T_{o}(M)$ with $\mathfrak{g}_{u}$. So we may write $\mathfrak{m}=T_{o}(M)^{c}$. Since $\left[\mathfrak{g}_{\alpha}, \mathfrak{g}_{\beta}\right] \in \mathfrak{g}_{\alpha+\beta}$ for $\alpha, \beta \in \Delta$, we see $[\mathfrak{f}, \mathfrak{m}] \subset \mathfrak{m}$ by the definition of $\Delta^{+}(\Phi)$. The complex structure $I$ of $M(\mathrm{~g}, \Phi)$ is given at $o$ by

$$
I\left(\mathrm{~A}_{\alpha}\right)=B_{\alpha}, I\left(B_{\alpha}\right)=-A_{\alpha} \quad \text { for } \alpha \in \Delta^{+}(\Phi) .
$$

Put $\mathfrak{m}^{ \pm}=\{X \in \mathfrak{m} ; I(X)= \pm \sqrt{-1} X\}$. Then we have

$$
\mathfrak{m}^{ \pm}=\sum_{\alpha \in \Delta+(\Phi)} \mathfrak{g}_{ \pm \alpha},
$$

and hence a direct sum $\mathfrak{m}=\mathfrak{m}^{+}+\mathfrak{m}^{-}$. An element of $\mathfrak{m}^{+}$is said to be of (1,0)-type. Now we define a mapping $p$ of $\Delta^{+}(\Phi)$ into $Z^{r}$ associated with $\Phi$ as follows: For $\alpha=\sum_{i=1}^{\ell} n_{i}(\alpha) \alpha_{i} \in \Delta^{+}\left(\alpha_{i_{1}}, \cdots, \alpha_{i_{r}}\right)$, we put 


$$
p_{\alpha}=\left(n_{i_{1}}(\alpha), \cdots, n_{i_{r}}(\alpha)\right) .
$$

This mapping $p$ plays an important role in this paper. Let $\omega^{\alpha}, \bar{\omega}^{\alpha}$ denote the dual forms of $E_{\alpha}, \bar{E}_{\alpha}\left(\alpha \in \Delta^{+}(\Phi)\right)$. Then any $G_{u}$-invariant Kählerian metric $g$ is given at $o$ by

$$
g=2 \sum_{\alpha \in \Delta+(\Phi)}\left(c \cdot p_{\alpha}\right) \omega^{\alpha} \cdot \bar{\omega}^{\alpha}
$$

for an $r$-tuple $c=\left(c_{1}, \cdots, c_{r}\right)$ of positive integers $c_{1}, \cdots, c_{r}$, where $c \cdot p_{\alpha}=$ $\sum_{a=1}^{r} c_{a} n_{i_{a}}(\alpha)$ (Borel [1] or Itoh [6]). Conversely, any bilinear form on $\mathfrak{m} \times \mathfrak{m}$ of this type can be extended to a $G_{u}$-invariant Kählerian metric on $M(\mathrm{~g}, \Phi)$.

When $\Delta^{+}(\Phi) \ni \alpha$ and $\beta$ satisfy $n_{i_{a}}(\alpha) \geqq n_{i_{a}}(\beta)$ for $a=1, \cdots, r$ and $n_{i_{a}}(\alpha)>n_{i_{a}}(\beta)$ for some $a$, we write $p_{\alpha}>p_{\beta}$. Hence we have equivalences $\alpha \in \Delta^{+}(\Phi) \Leftrightarrow E_{\alpha} \in \mathfrak{m}^{+} \Leftrightarrow p_{\alpha}>0$.

\section{§2. Covariant derivatives on Kählerian $C$-spaces}

In this section we consider a Kählerian $C$-space $(M(\mathfrak{g}, \Phi), g)$ constructed in Section 1, where $\Phi=\left\{\alpha_{i_{1}}, \cdots, \alpha_{i_{r}}\right\}$ is a non-empty subset of the fundamental root system $\Pi$ of a complex simple Lie algebra $g$ and $g$ is a $G_{u}$-invariant Kählerian metric on $M$ given by (1.8). We extend tensor fields, the connection and the connection form on $M$ naturally over $C$, and denote the extended ones by the same letters. Since $M$ is homogeneous Riemannian manifold, it is sufficient to consider the degree at the origin $o$.

First we have from (1.1) and (1.9)

$$
g\left(E_{\alpha}, E_{-\beta}\right)=-\left(c \cdot p_{\alpha}\right) B\left(E_{\alpha}, E_{-\beta}\right)=\left(c \cdot p_{\alpha}\right) \delta_{\alpha \beta} \quad \text { for } \alpha, \beta \in \Delta^{+}(\Phi) .
$$

When $r=1$, that is, $\Phi$ consists of a single element, we take a Kählerian metric $g$ such that $c=1$.

For $X \in \mathfrak{g}$ we denote by $X_{\mathrm{m}}$ (resp. $X_{\mathfrak{t}}$ ) the $\mathrm{m}$ (resp. $\mathfrak{f}$ )-component of $X$ with respect to the decomposition $g=\mathfrak{f}+\mathfrak{m}$. Define a symmetric bilinear mapping $U: \mathfrak{m} \times \mathfrak{m} \rightarrow \mathfrak{m}$ by

$$
2 g(U(X, Y), Z)=g\left([Z, X]_{\mathfrak{m}}, Y\right)+g\left(X,[Z, Y]_{\mathfrak{m}}\right) \quad \text { for } X, Y, Z \in \mathfrak{m} .
$$

Then the connection form $\Lambda: \mathfrak{m} \times \mathfrak{m} \rightarrow \mathfrak{m}$ associated with $g$ is given by (Kobayashi and Nomizu [17] and Nomizu [9])

$$
\Lambda(X) Y=U(X, Y)+\frac{1}{2}[X, Y] \quad \text { for } X, Y \in \mathfrak{m} .
$$


The curvature tensor $R$ of $g$ is given by (Nomizu [9])

$$
\begin{array}{r}
R(X, Y) Z=[\Lambda(X), \Lambda(Y)] Z-\Lambda\left([X, Y]_{\mathrm{m}}\right) Z-\left[[X, Y]_{\mathfrak{l}}, Z\right] \\
\text { for } X, Y, Z \in \mathfrak{m} .
\end{array}
$$

For $X \in \mathfrak{g}$ we denote by $X^{*}$ the vector field on $M$ induced by a oneparameter subgroup $\exp t X$ of $G_{u}$. It is easy to see that

$$
\left\{\begin{array}{l}
\left(X^{*}\right)_{o}=X_{\mathfrak{m}} \\
{\left[X^{*}, Y^{*}\right]=-[X, Y]^{*} \quad \text { for } X, Y \in \mathfrak{g} .}
\end{array}\right.
$$

The covariant derivative $\nabla_{X^{*}} Y^{*}$ of $Y^{*}$ in the direction $X^{*}$ is given at $o$ by ([7], p. 201)

$$
\left(\nabla_{X^{*}} Y^{*}\right)_{o}=\Lambda(Y) X=U(X, Y)-\frac{1}{2}[X, Y]_{\mathfrak{m}} \quad \text { for } X, Y \in \mathfrak{m} .
$$

In the following, in order to simplify the notation, we identify a root vector $E_{\alpha}$ with $\alpha$ itself for $\alpha \in \Delta$, and put $\nabla_{X} Y=\left(\nabla_{X^{*}} Y^{*}\right)_{0}$ for $X, Y \in \mathfrak{m}$. Under this identification, a subset $\Delta^{+}(\Phi) \cup \overline{\Delta^{+}(\Phi)}$ of $\Delta$ forms a basis of the complexified tangent space $\mathfrak{m}=T_{o}(M)^{C}$ of $M$ at $o$. We also call a root $\alpha \in \Delta^{+}(\Phi)$ a tangent vector.

M. Itoh [6] determined the connection form $\Lambda$ of $g$, which can be stated as

Proposition 2.1. Let $\alpha, \beta \in \Delta^{+}(\Phi)$. Then,

$$
\left\{\begin{array}{l}
\Lambda(\alpha) \beta=\left(c \cdot p_{\beta} / c \cdot p_{\alpha+\beta}\right)[\alpha, \beta], \\
\Lambda(\bar{\alpha}) \beta= \begin{cases}{[\bar{\alpha}, \beta]} & \text { if } p_{\alpha}<p_{\beta} \\
0 & \text { otherwise }\end{cases} \\
\Lambda(\alpha) \bar{\beta}= \begin{cases}{[\alpha, \bar{\beta}]} & \text { if } p_{\alpha}<p_{\beta} \\
0 & \text { otherwise. }\end{cases}
\end{array}\right.
$$

This and (2.6) give

Corollary 2.2. Let $\alpha, \beta \in \Delta^{+}(\Phi)$. Then,

$$
\left\{\begin{array}{l}
\nabla_{\alpha} \beta=-\left(c \cdot p_{\alpha} / c \cdot p_{\alpha+\beta}\right)[\alpha, \beta], \\
\nabla_{\alpha} \bar{\beta}= \begin{cases}-[\alpha, \bar{\beta}] & \text { if } p_{\alpha}>p_{\beta} \\
0 & \text { otherwise, }\end{cases} \\
\nabla_{\alpha} \beta= \begin{cases}-[\bar{\alpha}, \beta] & \text { if } p_{\alpha}>p_{\beta} \\
0 & \text { otherwise. }\end{cases}
\end{array}\right.
$$

The curvature tensor $R$ of $g$ can be restated as 


$$
\begin{aligned}
R(\alpha, \bar{\lambda}, \beta): & R(\alpha, \bar{\lambda}) \beta \\
& =[\Lambda(\alpha), \Lambda(\bar{\lambda})] \beta-\Lambda\left([\alpha, \bar{\lambda}]_{\mathrm{m}}\right) \beta-\left[[\alpha, \bar{\lambda}]_{t}, \beta\right] \\
& \quad \text { for } \alpha, \lambda, \beta \in \Delta^{+}(\Phi) .
\end{aligned}
$$

Put $R(\alpha, \bar{\lambda}, \beta, \bar{\mu})=g(R(\alpha, \bar{\lambda}, \beta), \bar{\mu})$. Then we obtain fundamental formulas

$$
\begin{aligned}
R(\alpha, \bar{\lambda}, \beta, \bar{\mu})=R(\beta, \bar{\lambda}, \alpha, \bar{\mu})=R(\alpha, \bar{\mu}, \beta, \bar{\lambda})= & \overline{R(\lambda, \bar{\alpha}, \mu, \bar{\beta})} \\
& \text { for } \alpha, \beta, \lambda, \mu \in \Delta^{+}(\Phi) .
\end{aligned}
$$

Now we define the $s$-th covariant derivative $\nabla^{s} R$ of $R$ inductively as follows:

$$
\begin{aligned}
R\left(\alpha, \bar{\lambda}, \beta ; \gamma_{1}, \cdots, \gamma_{s}\right)= & \sum_{\mu \in A^{+}(\Phi)} R\left(\alpha, \bar{\lambda}, \beta, \bar{\mu} ; \gamma_{1}, \cdots, \gamma_{s}\right) \mu / g(\mu, \bar{\mu}) \\
= & \nabla_{\gamma_{s}} R\left(\alpha, \bar{\lambda}, \beta ; \gamma_{1}, \cdots, \gamma_{s-1}\right) \\
& -R\left(\nabla_{\gamma_{s}} \alpha, \bar{\lambda}, \beta ; \gamma_{1}, \cdots, \gamma_{s-1}\right) \\
& -R\left(\alpha, \nabla_{r_{s}} \bar{\lambda}, \beta ; \gamma_{1}, \cdots, \gamma_{s-1}\right) \\
& -R\left(\alpha, \bar{\lambda}, \nabla_{\gamma_{s}} \beta ; \gamma_{1}, \cdots, \gamma_{s-1}\right) \\
& -\sum_{a=1}^{s-1} R\left(\alpha, \bar{\lambda}, \beta ; \gamma_{1}, \cdots, \nabla_{\gamma_{s}} \gamma_{a}, \cdots, \gamma_{s-1}\right) \\
& \quad \text { for } \alpha, \lambda, \beta \in \Delta^{+}(\Phi) \text { and } \gamma_{1}, \cdots, \gamma_{s} \in \mathbb{m} .
\end{aligned}
$$

When vectors $\gamma_{1}, \cdots, \gamma_{s}$ in (2.11) belong to $\Delta^{+}(\Phi)$, we write $\stackrel{+}{ }^{s} R$ instead of $\nabla^{s} R$, which is called the $s$-th covariant derivative of $(1,0)$-type of $R$. Then we have a basic property with respect to the covariant derivative of $R$ :

Lemma 2.3. For $\alpha, \lambda, \beta \in \Delta^{+}(\Phi)$ and $\gamma_{1}, \cdots, \gamma_{s} \in \mathfrak{m}$,

$$
R\left(\alpha, \bar{\lambda}, \beta ; \gamma_{1}, \cdots, \gamma_{s}\right) \in \mathfrak{g}_{\alpha-\lambda+\beta+\gamma_{1}+\cdots+\gamma_{s}} .
$$

Proof. This follows from a relation $\left[\mathfrak{g}_{\alpha}, \mathfrak{g}_{\beta}\right] \subset \mathfrak{g}_{\alpha+\beta},(2.7),(2.8)$ and (2.11).

Corollary 2.4. Let $\nu$ be the highest root in $\Delta$. Put $p_{\nu}=\left(n_{i_{1}}, \cdots, n_{i_{r}}\right)$, and $t=t(\Phi)=n_{i_{1}}+\cdots+n_{i_{r}}$. Then we have $\stackrel{\stackrel{D}{ }}{ }^{2 t-1} R=0$.

Proof. For $\delta \in \Delta^{+}(\Phi)$ we denote by $\|\delta\|$ the sum of the components of $p_{\delta}$. Then clearly, $1 \leqq\|\delta\| \leqq t=\|\nu\|$. Let $\alpha, \lambda, \beta, \gamma_{1}, \cdots, \gamma_{2 t-1} \in \Delta^{+}(\Phi)$. Then we have

$$
\begin{aligned}
& \left\|\alpha-\lambda+\beta+\gamma_{1}+\cdots+\gamma_{2 t-1}\right\| \\
& \quad=\|\alpha\|+\|\beta\|+\left\|\gamma_{1}\right\|+\cdots+\left\|\gamma_{2 t-1}\right\|-\|\lambda\| \\
& \quad \geqq 1+1+2 t-1-t=t+1 .
\end{aligned}
$$


This and the definition of $t$ show that a form $\alpha-\lambda+\beta+\gamma_{1}+\cdots+\gamma_{2 t-1}$ cannot be a root. Thus by Lemma 2.3 we have $R\left(\alpha, \bar{\lambda}, \beta ; \gamma_{1}, \cdots, \gamma_{2 t-1}\right)=$ 0 . q.e.d.

By Corollary 2.4, there exists uniquely the integer $d=d(\mathfrak{g}, \Phi)$ such

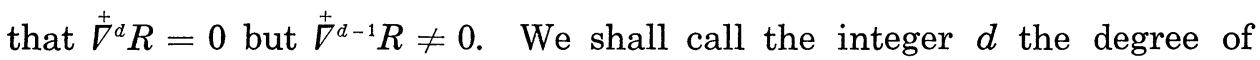
a $C$-space $M(\mathfrak{g}, \Phi)$. Hermitian symmetric spaces of compact type can be characterized as $C$-spaces with degree one. In fact, let $\Phi$ consist of a single element $\alpha_{a}$ such that $p_{\nu}=1$, where $\nu$ denotes the highest root in 4. Then Corollary 2.4 and (2.10) imply that a $C$-space $M\left(\mathfrak{g}, \alpha_{a}\right)$ is symmetric. Conversely, every irreducible Hermitian symmetric space of compact type can be expressed as a $C$-space of the form $M\left(\mathfrak{g}, \alpha_{a}\right)$ where $p_{\nu}=1$ (Wolf [13]).

\section{§3. C-spaces with degree two}

In this section we shall determine all irreducible $C$-spaces with degree two. Keep the notation in Section 2. Hereafter we denote by $\alpha, \beta, \gamma, \delta$, $\lambda, \mu$ any elements of $\Delta^{+}(\Phi)$ unless otherwise stated.

From (2.11) we have

$$
\begin{aligned}
R(\alpha, \bar{\lambda}, \beta ; \gamma, \delta)= & \nabla_{\delta} \nabla_{r} R(\alpha, \bar{\lambda}) \beta-\nabla_{\nabla_{\delta} \gamma} R(\alpha, \bar{\lambda}) \beta-\nabla_{\gamma} R\left(\nabla_{\delta} \alpha, \bar{\lambda}\right) \beta \\
& -\nabla_{\gamma} R\left(\alpha, \nabla_{\delta} \bar{\lambda}\right) \beta-\nabla_{r} R(\alpha, \bar{\lambda}) \nabla_{\delta} \beta \\
& -\nabla_{\delta} R\left(\nabla_{\gamma} \alpha, \bar{\lambda}\right) \beta+R\left(\nabla_{\nabla_{\delta} \gamma} \alpha, \bar{\lambda}\right) \beta+R\left(\nabla_{\gamma} \nabla_{\delta} \alpha, \bar{\lambda}\right) \beta \\
& +R\left(\nabla_{\gamma} \alpha, \nabla_{\delta} \bar{\lambda}\right) \beta+R\left(\nabla_{\gamma} \alpha, \bar{\lambda}\right) \nabla_{\delta} \beta \\
& -\nabla_{\delta} R\left(\alpha, \nabla_{\gamma} \bar{\lambda}\right) \beta+R\left(\nabla_{\delta} \alpha, \nabla_{\gamma} \bar{\lambda}\right) \beta+R\left(\alpha, \nabla_{\nabla_{\delta}} \bar{\lambda}\right) \beta \\
& +R\left(\alpha, \nabla_{\gamma} \nabla_{\delta} \bar{\lambda}\right) \beta+R\left(\alpha, \nabla_{r} \bar{\lambda}\right) \nabla_{\delta} \beta \\
& -\nabla_{\delta} R(\alpha, \bar{\lambda}) \nabla_{\gamma} \beta+R\left(\nabla_{\delta} \alpha, \bar{\lambda}\right) \nabla_{\gamma} \beta+R\left(\alpha, \nabla_{\delta} \bar{\lambda}\right) \nabla_{\gamma} \beta \\
& +R(\alpha, \bar{\lambda}) \nabla_{\nabla_{\delta} \gamma} \beta+R(\alpha, \bar{\lambda}) \nabla_{r} \nabla_{\delta} \beta .
\end{aligned}
$$

The equation (3.1) is not so complicated as it looks because it contains many simple cases. For example,

LEMma 3.1. Unless $p_{\alpha}>p_{\beta}$, then $\nabla_{\alpha} \bar{\beta}=0$ and $\Lambda(\bar{\beta}) \alpha=0$. In particular, if $\lambda$ is the highest root in $\Delta$, then $\nabla_{\alpha} \bar{\lambda}=0$ and $\Lambda(\bar{\lambda}) \alpha=0$.

Proof. This is a restatement of (2.7) and (2.8).

q.e.d.

Lemma 3.2. (1) If $\alpha+\beta-\lambda \notin \Delta$, then $R(\alpha, \bar{\lambda}) \beta=0$. (2) Let $\lambda$ satisfy $p_{\lambda} \geqq p_{r}$ for all $\gamma$. Let $\alpha$ and $\beta$ satisfy $p_{\lambda} \geqq p_{\alpha}+p_{\rho}$. Then $R(\alpha, \bar{\lambda}) \beta=0$. 
Proof. (1) is evident from a special case $R(\alpha, \bar{\lambda}) \beta \in \mathfrak{g}_{\alpha+\beta-\lambda}$ of Lemma 2.3. To show (2) we use (2.9). From Lemma 3.1 we have $\Lambda(\bar{\lambda}) \alpha=0$, and so from (2.7)

$$
\Lambda(\bar{\lambda}) \Lambda(\alpha) \beta \in \Lambda(\bar{\lambda}) \mathfrak{g}_{\alpha+\beta} .
$$

But, by Lemma 3.1, $\Lambda(\bar{\lambda}) \mathfrak{y}_{\alpha+\beta}=\{0\}$ whether $\alpha+\beta \in \Delta$ or not, hence $\Lambda(\bar{\lambda}) \Lambda(\alpha) \beta$ $=0$. If $\alpha+\bar{\lambda} \notin \Delta$, then $R(\alpha, \bar{\lambda}) \beta=0$ since $[\alpha, \bar{\lambda}]=0$. If $\alpha+\bar{\lambda} \in \Delta$, then $\lambda-\alpha \in \Delta^{+}(\Phi)$ since $p_{\lambda-\alpha} \geqq p_{\beta}>0$. Thus $R(\alpha, \bar{\lambda}) \beta=-\Lambda([\alpha, \bar{\lambda}]) \beta=0$ by Lemma 3.1. q.e.d.

Corollary 3.3 Let $\lambda, \alpha$ and $\beta$ satisfy at least one of the following two conditions:

(1) $\alpha+\beta-\lambda \notin \Delta$.

(2) $p_{\lambda} \geqq p_{\gamma}$ for all $\gamma$, and $p_{\lambda} \geqq p_{\alpha}+p_{\beta}$. Then,

$$
\begin{aligned}
R(\alpha, \bar{\lambda}, \beta ; \gamma, \delta)= & -\nabla_{\gamma} R\left(\nabla_{\delta} \alpha, \bar{\lambda}\right) \beta-\nabla_{\delta} R\left(\nabla_{\gamma} \alpha, \bar{\lambda}\right) \beta-\nabla_{r} R(\alpha, \bar{\lambda}) \nabla_{\delta} \beta \\
& -\nabla_{\delta} R(\alpha, \bar{\lambda}) \nabla_{r} \beta+R\left(\nabla_{\nabla_{\delta} \gamma} \alpha, \bar{\lambda}\right) \beta+R(\alpha, \bar{\lambda}) \nabla_{\nabla_{\delta} \gamma} \beta \\
& +R\left(\nabla_{\gamma} \nabla_{\delta} \alpha, \bar{\lambda}\right) \beta+R(\alpha, \bar{\lambda}) \nabla_{\gamma} \nabla_{\delta} \beta+R\left(\nabla_{r} \alpha, \bar{\lambda}\right) \nabla_{\delta} \beta \\
& +R\left(\nabla_{\delta} \alpha, \bar{\lambda}\right) \nabla_{\gamma} \beta .
\end{aligned}
$$

Proof. Apply (2.8), Lemma 3.1 and Lemma 3.2 (1) to (3.1). q.e.d.

Proposition 3.4. Let five vectors $\lambda, \alpha, \beta, \gamma, \delta \in \Delta^{+}(\Phi)$ satisfy the following three conditions:

(1) $\alpha+\beta-\lambda \notin \Delta$, or $p_{\lambda} \geqq p_{\gamma}$ for all $\gamma$ and $p_{\lambda} \geqq p_{\alpha}+p_{\beta}$ (the same condition as in Corollary 3.3).

(2) Let $\varepsilon$ be the sum of any three of $\alpha, \beta, \gamma, \delta$. Then $\varepsilon \notin \Delta$ and $p_{\varepsilon}>p_{\lambda}$.

(3) Let $\mu$ be the sum of any two of $\alpha, \beta, \gamma, \delta$. Then $[\mu, \bar{\lambda}] \in \mathfrak{f}$.

Then we have

$$
\begin{aligned}
2 R(\alpha, \bar{\lambda}, \beta, \bar{\lambda} ; \gamma, \delta)= & B([\delta,[\alpha, \bar{\lambda}]],[\beta,[\gamma, \bar{\lambda}]]) \\
& +B([\gamma,[\alpha, \bar{\lambda}]],[\beta,[\delta, \bar{\lambda}]]) \\
& -B([\alpha,[\gamma, \bar{\lambda}]],[\delta,[\beta, \bar{\lambda}]]) \\
& -B([\alpha,[\delta, \bar{\lambda}]],[\gamma,[\beta, \bar{\lambda}]]) .
\end{aligned}
$$

Proof. By Corollary 3.3 the equation (3.2) holds. The assumption (2) and (2.8) imply $\nabla_{\nabla_{\delta} \gamma} \alpha=\nabla_{\nabla_{\delta} \gamma} \beta=\nabla_{\gamma} \nabla_{\delta} \alpha=\nabla_{\gamma} \nabla_{\partial} \beta=0$. Thus,

$$
\begin{aligned}
\mathrm{A}:= & R(\alpha, \bar{\lambda}, \beta ; \gamma, \delta) \\
= & -\nabla_{\gamma} R\left(\nabla_{\delta} \alpha, \bar{\lambda}\right) \beta-\nabla_{\gamma} R(\alpha, \bar{\lambda}) \nabla_{\delta} \beta-\nabla_{\delta} R\left(\nabla_{\gamma} \alpha, \bar{\lambda}\right) \beta \\
& -\nabla_{\delta} R(\alpha, \bar{\lambda}) \nabla_{\gamma} \beta+R\left(\nabla_{\gamma} \alpha, \bar{\lambda}\right) \nabla_{\delta} \beta+R\left(\nabla_{\delta} \alpha, \bar{\lambda}\right) \nabla_{\gamma} \beta .
\end{aligned}
$$


We shall express the right hand side of (3.4) with respect to the bracket product [, ]. The assumptions (2) and (3), together with (2.7) and (2.8), imply $\left[\nabla_{\delta} \alpha, \bar{\lambda}\right] \in \mathfrak{f}, \Lambda(\bar{\lambda}) \beta=0$ and $\Lambda\left(\nabla_{\hat{o}} \alpha\right) \beta=0$. It follows from (2.8) and (2.9) that

$$
4 \nabla_{\gamma} R\left(\nabla_{\delta} \alpha, \bar{\lambda}\right) \beta=[\gamma,[[[\delta, \alpha], \bar{\lambda}], \beta]] .
$$

Applying similar argument to other five terms in (3.4), we find

$$
\begin{aligned}
4 A= & {[\gamma,[[[\delta, \alpha], \bar{\lambda}], \beta]]+[\delta,[[[\gamma, \alpha], \bar{\lambda}], \beta]]+[\gamma,[[\alpha, \bar{\lambda}],[\delta, \beta]]] } \\
& +[\delta,[[\alpha, \bar{\lambda}],[\gamma, \beta]]]-[[[\gamma, \alpha], \bar{\lambda}],[\delta, \beta]]-[[[\delta, \alpha], \bar{\lambda}],[\gamma, \beta]] .
\end{aligned}
$$

For a while, we write $\alpha \beta \gamma \delta$ for $[\alpha,[\beta,[\gamma,[\delta, \bar{\lambda}]]]]$. Then the Jacobi identities give

$$
\begin{aligned}
4 A= & (\gamma \beta \alpha \delta-\gamma \beta \delta \alpha)+(\gamma \alpha \beta \delta-\gamma \alpha \delta \beta) \\
& +\delta \beta \alpha \gamma-\delta \beta \gamma \alpha+\delta \alpha \beta \gamma-\delta \alpha \gamma \beta+\beta \delta \alpha \gamma-\delta \beta \alpha \gamma \\
& -\beta \delta \gamma \alpha+\delta \beta \gamma \alpha+\beta \gamma \alpha \delta-\gamma \beta \alpha \delta-\beta \gamma \delta \alpha+\gamma \beta \delta \alpha .
\end{aligned}
$$

In view of the identity $\gamma \alpha \beta \delta-\gamma \alpha \delta \beta=\beta \delta \gamma \alpha-\delta \beta \gamma \alpha$, obtained from $[\alpha,[\beta, \delta]]$ $=0$, we have

$$
4 A=-\gamma \beta \delta \alpha-\delta \beta \gamma \alpha+\beta \delta \alpha \gamma+\beta \gamma \alpha \delta .
$$

Now, by (2.1) and the subsequent comment and the formula $B([\alpha, \beta], \gamma)$ $=-B(\beta,[\alpha, \gamma])$, we have proposition.

q.e.d.

Here we shall specialize Proposition 3.4 in the form

Lemma 3.5. Assume that there exists uniquely a vector $\lambda \in \Delta^{+}(\Phi)$ admitting a decomposition of the form $\lambda=\sigma+\tau$ where $\sigma, \tau \in \Delta^{+}(\Phi)$. Assume that $(\sigma, \sigma)=(\tau, \tau)$ for all $\sigma, \tau \in \Delta^{+}(\Phi)$ such that $\lambda=\sigma+\tau$. Then, for all $\alpha, \beta, \gamma, \delta$ such that $2 \lambda=\alpha+\beta+\gamma+\delta$, the following holds:

$$
B([\alpha,[\beta, \bar{\lambda}]],[\gamma,[\delta, \bar{\lambda}]])=B([\beta,[\alpha, \bar{\lambda}]],[\delta,[\gamma, \bar{\lambda}]]) \text {. }
$$

Proof. From the assumption on $\lambda$ we have equivalences $\alpha+\beta \in \Delta \Leftrightarrow$ $\alpha+\beta=\lambda \Leftrightarrow \gamma+\delta=\lambda \Leftrightarrow \gamma+\delta \in \Delta$. Denote the left hand side of (3.5) by $\alpha \beta \cdot \gamma \delta$. If $\alpha+\beta \notin \Delta$, then we see $\alpha \beta \cdot \gamma \delta=\beta \alpha \cdot \delta \gamma$ since $[\alpha,[\beta, \bar{\lambda}]]=[\beta,[\alpha, \bar{\lambda}]]$ by Jacobi identity. If $\alpha+\beta \in \Delta$, then $\alpha+\beta=\gamma+\delta=\lambda$. Putting $[\alpha, \beta]$ $=N \lambda$ and $[\gamma, \delta]=M \lambda$, we see from (1.1) $[\beta, \bar{\lambda}]=N \bar{\alpha},[\bar{\lambda}, \alpha]=N \bar{\beta},[\delta, \bar{\lambda}]=$ $M \bar{\gamma}$ and $[\bar{\lambda}, \gamma]=M \bar{\delta}$. It follows that

$$
\begin{aligned}
\alpha \beta \cdot \gamma \delta-\beta \alpha \cdot \delta \gamma & =N M\left(B\left(H_{\alpha}, H_{\gamma}\right)-B\left(H_{\beta}, H_{\delta}\right)\right) \\
& =N M((\alpha, \gamma)-(\beta, \delta)) .
\end{aligned}
$$


On the other hand, taking the norm of $\alpha-\gamma=\delta-\beta$, we have $(\alpha, \alpha)-$ $2(\alpha, \gamma)+(\gamma, \gamma)=(\delta, \delta)-2(\delta, \beta)+(\beta, \beta)$. This and the assumption imply $(\alpha, \gamma)=(\delta, \beta)$.

q.e.d.

\section{§4. Theorems and proofs}

In this section we shall state our results and prove them.

THEOREM 4.1. Let $\alpha_{a}$ be any of the simple roots designed by the symbol $\otimes$ in the following Dynkin diagrams, and $g$ be a complex simple Lie algebra whose diagram contains $\alpha_{a}$. Then the degree of the irreducible $C$ space $M\left(\mathfrak{g}, \alpha_{a}\right)$ corresponding to the pair $\left(\mathfrak{g}, \alpha_{a}\right)$ is equal to 2.

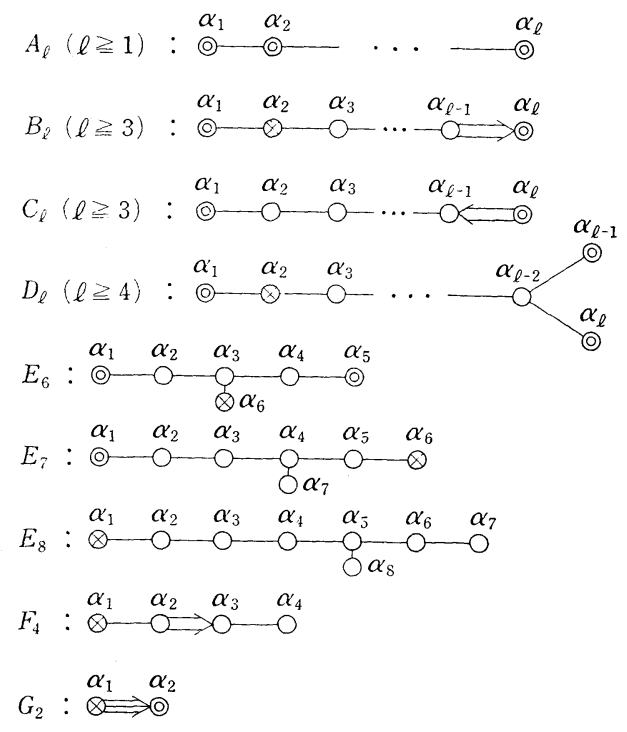

(In the diagrams, the double circle $\alpha_{a} \bigcirc$ means that the corresponding $C$ space $M\left(\mathfrak{g}, \alpha_{a}\right)$ is Hermitian symmetric.)

In order to prove Theorem 4.1 and for later use, we shall state here a positive root system $\Delta^{+}$of each complex simple Lie algebra $g$, a fundamental root system $\alpha_{1}, \cdots, \alpha_{\ell}$ of $\Delta^{+}$, and the subset $\Delta^{+}\left(\alpha_{a}\right)$ associated with a simple root $\alpha_{a}$ (cf. e.g. [3] or [4] appendix of [5]). For the five exceptional Lie algebras we omit $\Delta^{+}\left(\alpha_{a}\right)$ because the description is too complicated and we can do without them somehow.

$A_{\ell}(\ell \geqq 1): \quad$ A redundant orthonormal basis $\omega_{1}, \cdots, \omega_{\ell+1}$

with $\omega_{1}+\cdots+\omega_{\ell+1}=0$. 


$$
\Delta^{+}=\left\{\omega_{i}-\omega_{j}=\alpha_{i}+\cdots+\alpha_{j-1} ; 1 \leqq i<j \leqq \ell+1\right\} .
$$

For $1 \leqq a \leqq \ell$,

$$
\Delta^{+}\left(\alpha_{a}\right)=\left\{\omega_{i}-\omega_{j} ; i \leqq a \leqq j-1\right\} .
$$

$B_{\ell}(\ell \geqq 2)$ : An orthonormal basis $\omega_{1}, \cdots, \omega_{\ell}$.

$$
\begin{aligned}
\Delta^{+} & =\left\{\omega_{i}=\alpha_{i}+\cdots+\alpha_{\ell} ; 1 \leqq i \leqq \ell\right\} \\
& \cup\left\{\omega_{i}-\omega_{j}=\alpha_{i}+\cdots+\alpha_{j-1} ; 1 \leqq i<j \leqq \ell\right\} \\
& \cup\left\{\omega_{i}+\omega_{j}=\alpha_{i}+\cdots+\alpha_{j-1}+2 \alpha_{j}+\cdots+2 \alpha_{\ell} ; 1 \leqq i<j \leqq \ell\right\} .
\end{aligned}
$$

For $1 \leqq a \leqq \ell$,

$$
\begin{aligned}
\Delta^{+}\left(\alpha_{a}\right)= & \left\{\omega_{i} ; i \leqq a\right\} \cup\left\{\omega_{i}-\omega_{j} ; i \leqq a \leqq j-1\right\} \\
\cup & \left\{\omega_{i}+\omega_{j} ; i \leqq a, i<j\right\} .
\end{aligned}
$$

$C_{\ell}(\ell \geqq 3)$ : An orthonormal basis $\omega_{1}, \cdots, \omega_{\ell}$.

$$
\begin{aligned}
\Delta^{+} & =\left\{\omega_{i}=2 \alpha_{i}+\cdots+2 \alpha_{\ell-1}+\alpha_{\ell} ; 1 \leqq i \leqq \ell\right\} \\
& \cup\left\{\omega_{i}-\omega_{j}=\alpha_{i}+\cdots+\alpha_{j-1} ; 1 \leqq i<j \leqq \ell\right\} \\
& \cup\left\{\omega_{i}+\omega_{j}=\alpha_{i}+\cdots+\alpha_{j-1}+2 \alpha_{j}+\cdots+2 \alpha_{\ell-1}+\alpha_{\ell} ;\right. \\
\quad & 1 \leqq i<j \leqq \ell\} .
\end{aligned}
$$

For $1 \leqq a \leqq \ell$,

$$
\begin{aligned}
\Delta^{+}\left(\alpha_{a}\right)= & \left\{\omega_{i} ; i \leqq a\right\} \cup\left\{\omega_{i}-\omega_{j} ; i \leqq a \leqq j-1\right\} \\
\cup & \left\{\omega_{i}+\omega_{j} ; i \leqq a, i<j\right\} .
\end{aligned}
$$

$D_{\ell}(\ell \geqq 4): \quad$ An orthonormal basis $\omega_{1}, \cdots, \omega_{\ell}$.

$$
\begin{aligned}
\Delta^{+} & =\left\{\omega_{i}-\omega_{j}=\alpha_{i}+\cdots+\alpha_{j-1} ; 1 \leqq i<j \leqq \ell\right\} \\
& \cup\left\{\omega_{i}+\omega_{j}=\alpha_{i}+\cdots+\alpha_{j-1}+2 \alpha_{j}+\cdots+2 \alpha_{\ell-2}+\alpha_{\ell-1}+\alpha_{\ell} ;\right. \\
& \quad 1 \leqq i<j \leqq \ell-1\} \\
& \cup\left\{\omega_{i}+\omega_{\ell}=\alpha_{i}+\cdots+\alpha_{\ell-2}+\alpha_{\ell} ; 1 \leqq i \leqq \ell-1\right\} .
\end{aligned}
$$

For $1 \leqq a \leqq \ell-2$,

$$
\begin{aligned}
& \Delta^{+}\left(\alpha_{a}\right)=\left\{\omega_{i}-\omega_{j} ; i \leqq a \leqq j-1\right\} \\
& \cup\left\{\omega_{i}+\omega_{j} ; i \leqq a, i<j\right\} .
\end{aligned}
$$

$E_{6}$ : A basis $\omega_{1}, \cdots, \omega_{6}$ with $\left(\omega_{i}, \omega_{i}\right)=8$ and $\left(\omega_{i}, \omega_{j}\right)=-1$ for $i \neq j$.

$$
\begin{aligned}
& \Delta^{+}=\left\{\omega_{i}-\omega_{j} ; 1 \leqq i<j \leqq 6\right\} \\
& \cup\left\{\omega_{i}+\omega_{j}+\omega_{k} ; 1 \leqq i<j<k \leqq 6\right\} \cup\left\{\omega_{1}+\cdots+\omega_{6}\right\} . \\
& \alpha_{i}=\omega_{i}-\omega_{i+1}(1 \leqq i \leqq 5) \text { and } \alpha_{6}=\omega_{4}+\omega_{5}+\omega_{6} .
\end{aligned}
$$


$E_{7}:$ A basis $\omega_{1}, \cdots, \omega_{7}$ with $\left(\omega_{i}, \omega_{i}\right)=8$ and $\left(\omega_{i}, \omega_{j}\right)=-1$ for $i \neq j$.

$$
\begin{aligned}
& \Delta^{+}=\left\{\omega_{i}-\omega_{j} ; 1 \leqq i<j \leqq 7\right\} \\
& \cup\left\{\omega_{i}+\omega_{j}+\omega_{k} ; 1 \leqq i<j<k \leqq 7\right\} \\
& \cup\left\{\omega_{1}+\cdots+\hat{\omega}_{i}+\cdots+\omega_{7} ; 1 \leqq i \leqq 7\right\} \\
& \alpha_{i}=\omega_{i}-\omega_{i+1}(1 \leqq i \leqq 6) \text { and } \alpha_{7}=\omega_{5}+\omega_{6}+\omega_{7} .
\end{aligned}
$$

$E_{8}$ : A basis $\omega_{1}, \cdots, \omega_{8}$ with $\left(\omega_{i}, \omega_{i}\right)=8$ and $\left(\omega_{i}, \omega_{j}\right)=-1$ for $i \neq j$.

$$
\begin{aligned}
& \Delta^{+}=\left\{\omega_{i}-\omega_{j} ; 1 \leqq i<j \leqq 8\right\} \\
& \cup\left\{\omega_{i}+\omega_{j}+\omega_{k} ; 1 \leqq i<j<k \leqq 8\right\} \\
& \cup\left\{\omega_{1}+\cdots+\hat{\omega}_{i}+\cdots+\hat{\omega}_{j}+\cdots+\omega_{8} ; 1 \leqq i<j \leqq 8\right\} \\
& \cup\left\{\omega_{1}+\cdots+\omega_{i-1}+2 \omega_{i}+\omega_{i+1}+\cdots+\omega_{8} ; 1 \leqq i \leqq 8\right\} \\
& \alpha_{i}=\omega_{i}-\omega_{i+1}(1 \leqq i \leqq 7) \text { and } \alpha_{8}=\omega_{6}+\omega_{7}+\omega_{8} .
\end{aligned}
$$

$F_{4}:$ An orthonormal basis $\omega_{1}, \omega_{2}, \omega_{3}, \omega_{4}$.

$$
\begin{aligned}
\Delta^{+} & =\left\{\omega_{i} \pm \omega_{j} ; 1 \leqq i<j \leqq 4\right\} \\
& \cup\left\{\omega_{i} ; 1 \leqq i \leqq 4\right\} \\
& \cup\left\{\frac{1}{2} \omega_{1} \pm \frac{1}{2} \omega_{2} \pm \frac{1}{2} \omega_{3} \pm \frac{1}{2} \omega_{4} ; \text { independent signs }\right\} \\
\alpha_{1}= & \omega_{2}-\omega_{3}, \alpha_{2}=\omega_{3}-\omega_{4}, \alpha_{3}=\omega_{4} \text { and } \alpha_{4}=\frac{1}{2}\left(\omega_{1}-\omega_{2}-\omega_{3}-\omega_{4}\right) .
\end{aligned}
$$

$G_{2}:$ A redundant basis $\omega_{1}, \omega_{2}, \omega_{3}$ with $\omega_{1}+\omega_{2}+\omega_{3}=0,\left(\omega_{i}, \omega_{i}\right)=2$ and $\left(\omega_{i}, \omega_{j}\right)=-1$ for $i \neq j$.

$$
\begin{aligned}
& \Delta^{+}=\left\{\omega_{i} ; 1 \leqq i \leqq 3\right\} \cup\left\{\omega_{i}-\omega_{j} ; 1 \leqq i<j \leqq 3\right\} \\
& \alpha_{1}=\omega_{1}-\omega_{2}, \quad \alpha_{i}=-\omega_{1} .
\end{aligned}
$$

Proof of Theorem 4.1. We use Proposition 3.4 and Lemma 3.5. First, we assert that $\Delta^{+}\left(\alpha_{a}\right)$ satisfies the condition of Lemma 3.5. In fact, when $\mathrm{g}=E_{\ell}(\ell=6,7,8)$, it is trivial since all roots have the same length. In other cases, let $\lambda$ be the highest root in $\Delta$. Then $\lambda \in \Delta^{+}\left(\alpha_{a}\right)$. Furthermore, it can be easily checked that $p_{\lambda}=2$ and $p_{\varepsilon}=1$ for any $\varepsilon \in \Delta^{+}\left(\alpha_{a}\right)$ $-\{\lambda\}$. Here, we write out all possible decompositions $\lambda=\alpha+\beta, \alpha, \beta \epsilon$ $\Delta^{+}\left(\alpha_{a}\right)$ of $\lambda$ for $\mathrm{g}=B_{\ell}, D_{\ell}, F_{4}$ and $G_{2}$.

$$
\begin{aligned}
& B_{\ell}(\ell \geqq 3): \quad \lambda=\omega_{1}+\omega_{2}=\left(\omega_{1} \pm \omega_{j}\right)+\left(\omega_{2} \mp \omega_{j}\right) \quad(3 \leqq j \leqq \ell) . \\
& D_{\ell}(\ell \geqq 4): \quad \lambda=\omega_{1}+\omega_{2}=\left(\omega_{1} \pm \omega_{j}\right)+\left(\omega_{2} \mp \omega_{j}\right) \quad(3 \leqq j \leqq \ell) . \\
& F_{4}: \lambda=\omega_{1}+\omega_{2}=\left(\omega_{1} \pm \omega_{j}\right)+\left(\omega_{2} \mp \omega_{j}\right) \quad(j=3,4) \\
& \quad=\frac{1}{2}\left(\omega_{1}+\omega_{2} \pm \omega_{3} \pm \omega_{4}\right)+\frac{1}{2}\left(\omega_{1}+\omega_{2} \mp \omega_{3} \mp \omega_{4}\right) . \\
& G_{2}: \lambda=\omega_{3}-\omega_{2}=\left(\omega_{3}-\omega_{1}\right)+\left(\omega_{1}-\omega_{2}\right) .
\end{aligned}
$$


As a result, we find $(\alpha, \alpha)=(\beta, \beta)$ in each case, which proves our assertion.

Next, we assert that $R(\alpha, \bar{\varepsilon}, \beta ; \gamma, \delta)=0$ for any $\alpha, \beta, \gamma, \delta, \varepsilon \in \Delta^{+}\left(\alpha_{a}\right)$. To show this, put $\mu=\alpha+\beta+\gamma+\delta-\varepsilon$. If $\mu \notin \Delta$, then Lemma 2.3 proves our assertion. So, assume $\mu \in \Delta$. In view of the fact $p_{\nu}=1$ or 2 for any $\nu$, we have $\mu=\varepsilon=\lambda$ and $p_{a}=p_{\beta}=p_{\gamma}=p_{\delta}=1$. Then it can be easily seen that our five vectors $\lambda, \alpha, \beta, \gamma$ and $\delta$ satisfy the condition of Proposition 3.4. This, together with Lemma 3.5, completes the proof of the Theorem.

q.e.d.

Along the same line, we can prove

Theorem 4.2. The degree of an irreducible $C$-space $M\left(A_{\ell}, \alpha_{1}, \alpha_{\ell}\right)(\ell \geqq 2)$. is equal to 2.

Proof. Since $\Delta^{+}(\Phi)=\left\{\alpha_{1}+\cdots+\alpha_{i} ; 1 \leqq i \leqq \ell\right\} \cup\left\{\alpha_{j}+\cdots+\alpha_{\ell} ; 1 \leqq\right.$ $j \leqq \ell\}$, the highest root $\lambda=\omega_{1}-\omega_{\ell+1}=\alpha_{1}+\cdots+\alpha_{\ell}$ is the only vector in $\Delta^{+}(\Phi)$ admitting a decomposition of the form $\lambda=\varepsilon_{1}+\varepsilon_{2}$ where $\varepsilon_{1}, \varepsilon_{2} \in$ $\Delta^{+}(\Phi)$. Moreover, all elements of $\Delta$ have the same length. Thus $\lambda$ satisfies the condition of Lemma 3.5.

In view of the fact that $p_{\lambda}=(1,1)$ and $p_{\alpha}=(1,0)$ or $(0,1)$ when $\alpha \epsilon$ $\Delta^{+}(\Phi)-\{\lambda\}$, we see that, if six vectors $\alpha, \beta, \gamma, \delta, \varepsilon, \mu \in \Delta^{+}(\Phi)$ satisfy $\varepsilon+\mu$ $=\alpha+\beta+\gamma+\delta$, then $\varepsilon=\mu=\lambda$ and the sum of two of $\alpha, \beta, \gamma, \delta$ must be equal to $\lambda$, and hence $\lambda, \alpha, \beta, \gamma$ and $\delta$ satisfy the condition of Proposition 3.4. Now, Proposition 3.4 and Lemma 3.4 imply $R(\alpha, \bar{\lambda}, \beta ; \gamma, \delta)=0$. q.e.d.

In the following, in order to prove that the $C$-spaces of eight types given in Theorems 4.1 and 4.2 exhaust all irreducible $C$-spaces with degree two, we shall give a sufficient condition for a $C$-space $M(\mathfrak{g}, \Phi)$ to satisfy $\stackrel{+}{\nabla}^{2} R \neq 0$.

LEMMA 4.3. Let $\alpha, \beta, \gamma \in \Delta^{+}(\Phi)$ satisfy

(1) $\alpha+\beta \in \Delta$,

(2) $\beta-\gamma \in \Delta^{+}$,

(3) $\beta+\gamma \notin \Delta$,

(4) $\alpha+\beta-\gamma \notin \Delta$,

(5) $\alpha+\beta+\gamma \notin \Delta$. Then,

$$
\begin{aligned}
R(\alpha, \bar{\lambda}, \beta ; \gamma, \alpha)= & -\nabla_{\gamma} R\left(\alpha, \bar{\lambda}, \nabla_{\alpha} \beta\right)-\nabla_{\alpha} R\left(\nabla_{\gamma} \alpha, \bar{\lambda}, \beta\right) \\
& +R\left(\nabla_{\nabla_{\alpha \gamma}} \alpha, \bar{\lambda}, \beta\right)+R\left(\nabla_{\gamma} \alpha, \bar{\lambda}, \nabla_{\alpha} \beta\right),
\end{aligned}
$$

where we put $\lambda=\alpha+\beta$. 
Proof. Put $\delta=\alpha$ in (3.1). Then, by (2.8), all terms except for four ones in (4.1) vanish since $R(\alpha, \bar{\lambda}, \beta)=0$ by Lemma $3.2, \nabla_{\alpha} \alpha=\nabla_{\alpha} \bar{\lambda}=0$ by Lemma $3.1, \nabla_{\nabla_{\alpha} \gamma} \bar{\lambda}=0$ by (2), $\nabla_{\gamma} \beta=0$ by (3), $\nabla_{\gamma} \bar{\lambda}=0$ by (4), and $\nabla_{\nabla_{\alpha} \gamma} \beta=$ $\nabla_{\gamma} \nabla_{\alpha} \beta=0$ by (5).

q.e.d.

LEMMA 4.4. Let $\alpha, \beta, \gamma \in \Delta^{+}(\Phi)$ satisfy $(1) \sim(5)$ in Lemma 4.3 and in addition the following (6)', (7) and (8).

(6)' $2 \alpha+\gamma \notin \Delta$ or $\alpha+\gamma-\beta \notin \Delta^{+}(\Phi)$,

(7) $2 \alpha+\beta \notin \Delta$,

(8) $2 \alpha+\beta+\gamma \notin \Delta$. Then,

$$
\begin{aligned}
R(\alpha, \bar{\lambda}, \beta ; \gamma, \alpha)= & b(b+1)[\alpha,[[\alpha, \gamma], \bar{\lambda}], \beta] \\
& -b\left[\left[\nabla_{[\alpha, \gamma]} \alpha, \bar{\lambda}\right], \beta\right],
\end{aligned}
$$

where we put $\lambda=\alpha+\beta$ and $b=-c \cdot p_{\alpha} / c \cdot p_{\alpha+\gamma}$.

Proof. We shall deform the right hand side of (4.1) using (2.9) and Lemma 3.1;

$$
\begin{aligned}
& R(\alpha, \bar{\lambda}) \nabla_{\alpha} \beta=-\Lambda([\alpha, \bar{\lambda}]) \nabla_{\alpha} \beta \quad \text { (by (7)) } \\
& =-\left[[\alpha, \bar{\lambda}], \nabla_{\alpha} \beta\right] \quad(\text { by }(2.7)), \\
& R\left(\nabla_{\alpha} \alpha, \bar{\lambda}\right) \beta=-\Lambda\left(\left[\nabla_{\gamma} \alpha, \bar{\lambda}\right]_{\mathfrak{m}}\right) \beta \\
& -\left[\left[\nabla_{r} \alpha, \bar{\lambda}\right]_{\mathfrak{t}}, \beta\right] \quad \text { (by (2) and (8)) } \\
& =-\left[\left[\nabla_{r} \alpha, \bar{\lambda}\right], \beta\right] \quad \text { (by (2.7)), } \\
& R\left(\nabla_{\gamma} \alpha, \bar{\lambda}\right) \nabla_{\alpha} \beta=-\Lambda\left(\left[\nabla_{\gamma} \alpha, \bar{\lambda}\right]\right) \nabla_{\alpha} \beta \\
& -\left[\left[\nabla_{\gamma} \alpha, \bar{\lambda}\right]_{t}, \beta\right] \quad \text { (by (2) and (8)) } \\
& =-\left[\left[\nabla_{\gamma} \alpha, \bar{\lambda}\right], \nabla_{\alpha} \beta\right] \quad(\text { by }(2.7)), \\
& R\left(\nabla_{\nabla_{\alpha \gamma}} \alpha, \bar{\lambda}\right) \beta=-\Lambda\left(\left[\nabla_{\nabla_{\alpha \gamma}} \alpha, \bar{\lambda}\right]_{\mathrm{m}}\right) \beta \\
& -\left[\left[\nabla_{\nabla_{\alpha \gamma}} \alpha, \bar{\lambda}\right]_{t}, \beta\right] \quad \text { (by (8)) } \\
& =-\left[\left[\nabla_{\nabla_{\alpha \gamma}} \alpha, \bar{\lambda}\right], \beta\right] \quad \text { (by (6) and (2.7)) . }
\end{aligned}
$$

Here, put $a=-c \cdot p_{\alpha} / c \cdot p_{\alpha+\beta}$. Then $\nabla_{\alpha} \beta=a[\alpha, \beta]$ and $\nabla_{\beta} \alpha=(a+1)[\alpha, \beta]$ by (2.8). On the other hand, since $\nabla_{\gamma} \alpha=(b+1)[\alpha, \gamma]$, we have

$$
\begin{aligned}
R(\alpha, \bar{\lambda}, \beta ; \gamma, \alpha)= & a(b+1)[[[\alpha, \bar{\lambda}],[\alpha, \beta]], \gamma] \\
& +b(b+1)[\alpha,[[[\alpha, \gamma], \bar{\lambda}], \beta] \\
& -a(b+1)[[[\alpha, \gamma], \bar{\lambda}],[\alpha, \beta]] \\
& -\left[\left[\nabla_{\nabla_{\alpha \gamma}} \alpha, \bar{\lambda}\right], \beta\right] .
\end{aligned}
$$

The sum of the first and third terms vanishes because of (4), (5) and Jacobi identity. q.e.d. 
Lemma 4.5. Let $\alpha, \beta, \gamma \in \Delta^{+}(\Phi)$ satisfy (1) (5) in Lemma 4.3 and (7), (8) in Lemma 4.4 and in addition the following (6), (9)

(6) $2 \alpha+\gamma \notin \Delta$,

(9) $\alpha+\gamma \in \Delta$. Then,

$$
R(\alpha, \overline{\alpha+\beta}, \beta ; \gamma, \alpha)=b(b+1)[\alpha,[[[\alpha, \gamma], \overline{\alpha+\beta}], \beta]] \neq 0 .
$$

Proof. This is obtained from Lemma 4.4 and the fact that $\alpha+\gamma \in \Delta$, $\gamma-\beta \in \Delta$ and $b(b+1) \neq 0$.

q.e.d.

Remark 4.6. (1) If $\alpha+\beta$ is the highest root in $\Delta$, then the conditions (5), (7) and (8) are necessarily satisfied. (2) Let $\Phi^{\prime}$ be another subset of a fundamental root system $\left\{\alpha_{1}, \cdots, \alpha_{\ell}\right\}$ of $\mathrm{g}$, and $R^{\prime}$ denote the curvature tensor of any Kählerian metric of the form (1.9) on a $C$-space $M\left(\mathfrak{g}, \Phi^{\prime}\right)$. Assume that $\Phi \subset \Phi^{\prime}$ and $\alpha, \beta, \gamma \in \Delta^{+}(\Phi)$ satisfy the condition of Lemma 4.5. Then Lemma 4.5 implies

$$
R(\alpha, \overline{\alpha+\beta}, \beta ; \gamma, \alpha)=R^{\prime}(\alpha, \overline{\alpha+\beta}, \beta ; \gamma, \alpha)
$$

ThEOREm 4.7. Let $\mathrm{g}$ be a complex simplex Lie algebra and $\Phi$ be $a$ non-empty subset of a fundamental root system of g. Assume that the $C$ space $M(\mathfrak{g}, \Phi)$ corresponding to the pair $(\mathfrak{g}, \Phi)$ is neither a Hermitian symmetric space nor any of the C-space of eight types given in Theorems 4.1 and 4.2. Then, the degree $d$ of the $C$-space $M(\mathfrak{g}, \Phi)$ is not smaller than 3. If $\Phi$ consists of a single element $\alpha_{a}$ and the coefficient of $\alpha_{a}$ in the highest root in $\Delta$ is equal to 2 , then $d=3$.

Proof. Throughout the proof, let $\alpha_{a}$ stand for any of the simple roots designed by $\bigcirc$ in the diagrams of Theorem 4.1 (thus not a simple root designed by (O) or $\otimes$ ). We divide the proof into four parts;

(I) Case where $\Phi=\left\{\alpha_{a}\right\}$. We shall show $d=3$. Let $\lambda$ be the highest root in $\Delta$. Then, since $p_{\lambda}=2$, we have $d \leqq 3$ by Corollary 3.4. Thus, by Corollary 4.5 it suffices to find three roots $\alpha, \beta$ and $\gamma$ in $\Delta^{+}\left(\alpha_{a}\right)$ satisfying nine conditions $(1) \sim(9)$ in Lemmas 3.3, 3.4 and 3.5. In the following we state examples of such roots $\alpha, \beta$ and $\gamma$.

(A) For $\mathfrak{g}=B_{\ell}(\ell \geqq 4,3 \leqq a<\ell-1)$ or $D_{\ell}(\ell \geqq 5,3 \leqq a \leqq \ell-1)$, put $\alpha=\omega_{1}+\omega_{\ell}, \beta=\omega_{2}-\omega_{\ell}$ and $\gamma=\omega_{a}-\omega_{\ell}$.

(B) For $g=C_{\ell}(\ell \geqq 3,2 \leqq a \leqq \ell-1)$, put $\alpha=\omega_{1}+\omega_{\ell}, \beta=\omega_{1}-\omega_{\ell}$ and $\gamma=\omega_{a}-\omega_{\ell}$.

(C) For $\mathfrak{g}=E_{6}$, put 


$$
\begin{aligned}
& \alpha=\left(\begin{array}{lllll}
0 & 1 & 2 & 1 & 1 \\
& & 1 &
\end{array}\right)=\omega_{2}+\omega_{3}+\omega_{5}, \\
& \beta=\left(\begin{array}{lllll}
1 & 1 & 1 & 1 & 0
\end{array}\right)=\omega_{1}+\omega_{4}+\omega_{6},
\end{aligned}
$$

and

$$
\gamma=\left(\begin{array}{lllll}
1 & 1 & 1 & 1 & 0 \\
& & 0 & &
\end{array}\right)=\omega_{1}-\omega_{5}
$$

where $\left(\begin{array}{lllll}n_{1} & n_{2} & n_{3} & n_{4} & n_{5} \\ & & n_{6} & & \end{array}\right)$ means a root $n_{1} \alpha_{1}+\cdots+n_{6} \alpha_{8}$. Hereafter we use the similar notation.

(D) For $\mathfrak{g}=E_{7}$, put

$$
\begin{aligned}
& \alpha=\left(\begin{array}{llllll}
0 & 1 & 1 & 2 & 1 & 1 \\
& & & 1 &
\end{array}\right)=\omega_{2}+\omega_{4}+\omega_{6}, \\
& \beta=\left(\begin{array}{llllll}
1 & 1 & 2 & 2 & 2 & 1 \\
& & & 1 &
\end{array}\right)=\omega_{1}+\omega_{3}+\omega_{5},
\end{aligned}
$$

and

$$
\gamma=\left(\begin{array}{llllll}
1 & 1 & 2 & 2 & 1 & 0 \\
& & & 1 & &
\end{array}\right)=\omega_{1}+\omega_{3}+\omega_{7}
$$

(E) For $\mathfrak{g}=E_{8}$, put

$$
\begin{aligned}
& \alpha=\left(\begin{array}{lllllll}
1 & 1 & 1 & 1 & 1 & 1 & 1 \\
& & & & 1 & &
\end{array}\right)=\omega_{1}+\omega_{6}+\omega_{7}, \\
& \beta=\left(\begin{array}{lllllll}
1 & 2 & 3 & 4 & 5 & 3 & 1
\end{array}\right)=\omega_{1}+\cdots+\omega_{5}+\omega_{8},
\end{aligned}
$$

and

$$
\gamma=\left(\begin{array}{rrrrrrr}
0 & 1 & 2 & 3 & 4 & 2 & 1 \\
& & & & 2 & &
\end{array}\right)=\omega_{2}+\cdots+\omega_{5}+\omega_{7}+\omega_{8} .
$$

(F) For $\mathfrak{g}=F_{4}$, put

$$
\begin{aligned}
& \alpha=\left(\begin{array}{lll}
1 & 1 \Rightarrow 1 & 1
\end{array}\right), \\
& \beta=\left(\begin{array}{lll}
1 & 2 \Rightarrow 3 & 1
\end{array}\right),
\end{aligned}
$$

and

$$
\gamma=\left(\begin{array}{lll}
0 & 1 \Rightarrow 2 & 1
\end{array}\right) .
$$

(II) Case where $\alpha_{a} \in \Phi$. By Lemma 4.5 and Remark 4.6 (2) we have $R(\alpha, \overline{\alpha+\beta}, \beta ; \gamma, \alpha) \neq 0$ for $\alpha, \beta$ and $\gamma$ just given in the case (I). 
(III) Case where $\Phi$ consists of only simple roots designed by (O) or $\otimes$, and where $g \neq B_{2}$. We shall describe all possible pairs $(g, \Phi)$ and examples of three roots $\alpha, \beta$ and $\gamma$ in $\Delta^{+}(\Phi)$ satisfying nine conditions (1) $\sim(9)$.

(A) For $\left(A_{\ell}, \alpha_{r}, \alpha_{s}\right)(\ell \geqq 3,1 \leqq r<s<\ell+1)$, put $\alpha=\omega_{1}-\omega_{r+1}, \beta=$ $\omega_{r+1}-\omega_{\ell+1}$ and $\gamma=\omega_{r+1}-\omega_{s+1}$.

(B) For $\left(B_{\ell}, \alpha_{1}, \alpha_{2}\right)(\ell \geqq 3)$ or $\left(D_{\ell}, \alpha_{1}, \alpha_{2}\right)(\ell \geqq 4)$, put $\alpha=\omega_{2}-\omega_{3}, \beta=$ $\omega_{1}+\omega_{3}$ and $\gamma=\omega_{1}-\omega_{2}$.

(C) For $\left(B_{\ell}, \alpha_{1}, \alpha_{\ell}\right)(\ell \geqq 3)$ or $\left(B_{\ell}, \alpha_{2}, \alpha_{\ell}\right)(\ell \geqq 3)$, put $\alpha=\omega_{1}-\omega_{\ell}, \beta=$ $\omega_{2}+\omega_{\ell}$ and $\gamma=\omega_{\ell}$.

(D) For $\left(D_{\ell}, \alpha_{1}, \alpha_{\ell-1}\right)(\ell \geqq 4)$ or $\left(D_{\ell}, \alpha_{2}, \alpha_{\ell}\right)(\ell \geqq 4)$, put $\alpha=\omega_{1}+\omega_{\ell}, \beta=$ $\omega_{2}-\omega_{\ell}$ and $\gamma=\omega_{a}-\omega_{\ell}$.

(E) For $\left(D_{\ell}, \alpha_{\ell-1}, \alpha_{\ell}\right)(\ell \geqq 4)$, put $\alpha=\omega_{\ell-3}-\omega_{\ell}, \beta=\omega_{\ell-2}+\omega_{\ell}$ and $\gamma=$ $\omega_{\ell-1}+\omega_{\ell}$.

(F) For $\left(C_{\ell}, \alpha_{1}, \alpha_{\ell}\right)(\ell \geqq 3)$, put $\alpha=\omega_{1}-\omega_{\ell}, \beta=\omega_{1}+\omega_{\ell}$ and $\gamma=\omega_{2}$ $+\omega_{\ell}$.

(G) For $\left(E_{6}, \alpha_{1}, \alpha_{5}\right)$ or $\left(E_{6}, \alpha_{1}, \alpha_{6}\right)$, take the same $\alpha, \beta$ and $\gamma$ as in the subcase (C) of the case (I). By the symmetry of the Dynkin diagram of $E_{6}$, we can find similar three roots for $\left(E_{6}, \alpha_{5}, \alpha_{6}\right)$.

(H) For $\left(E_{7}, \alpha_{1}, \alpha_{6}\right)$, take the same $\alpha, \beta$ and $\gamma$ as in the subcase (D) of the case (I).

(IV) Case where $\mathfrak{g}=B_{2}$ and $\Phi=\left\{\alpha_{1}, \alpha_{2}\right\}$. Put $\alpha=\omega_{2}, \beta=\omega_{1}, \gamma=\omega_{1}-\omega_{2}$ and $\delta=\omega_{1}+\omega_{2}$. Then we can take them in such a way that $[\alpha, \gamma]=\beta$ and $[\alpha, \beta]=\delta$, and so $[\bar{\alpha}, \beta]=-\gamma,[\bar{\delta}, \beta]=\bar{\alpha}$ and $[\bar{\alpha}, \delta]=\beta$ by (1.1) (cf. e.g. [4], p. 277). On the other hand, since three roots $\alpha, \beta$ and $\gamma$ satisfy the condition of Lemma 4.4, we have

$$
\begin{aligned}
R(\alpha, \bar{\delta}, \beta ; \gamma, \alpha)= & b(b+1)[\alpha,[[[\alpha, \gamma], \bar{\delta}], \beta]] \\
& -b\left[\left[\nabla_{\beta} \alpha, \bar{\delta}\right], \beta\right] .
\end{aligned}
$$

Here, from (2.7) we have $\nabla_{\beta} \alpha=a[\beta, \alpha]=a \delta$, where $a=-c \cdot p_{\beta} / c \cdot p_{\beta+\alpha}$. Hence,

$$
\begin{aligned}
R(\alpha, \bar{\delta}, \beta ; \gamma, \alpha) & =b(b+1)[\alpha,[[\beta, \bar{\delta}], \beta]]-a b[[\delta, \delta], \beta] \\
& =b(a+b+1) \beta .
\end{aligned}
$$

But, in view of $p_{\alpha}=(0,1), p_{\beta}=(1,1), p_{\gamma}=(1,0)$ and $c=\left(c_{1}, c_{2}\right)$, we have $a+b+1=-c_{2}^{2} /\left(c_{1}+c_{2}\right)\left(c_{1}+2 c_{2}\right) \neq 0$, which completes the proof of Theorem 4.7 . 


\section{REFERENCES}

[1] A. Borel, Kählerian coset spaces of semi-simple Lie groups, Proc. Nat. Acad. Sci. U. S. A., 40 (1954), 1147-1151.

[2] A. Borel and F. Hirzebruch, Characteristic classes and homogeneous spaces I, Amer. J. Math., 80 (1958), 458-538.

[ 3 ] N. Bourbaki, Groups et algebres de Lie, IV, V et VI, Hermann, Paris, 1968.

[ 4 ] E. Cartan, Les groupes reels simples, finis et continus, Euvres Completes, Partie I, Volume 1 (1952), 399-491.

[5] Hans Freudenthal and H. de Vries, Linear Lie Groups, Academic Press, 1969.

[6] M. Itoh, On curvature properties of Kähler $C$-spaces, J. Math. Soc. Japan, 30 (1976), 39-71.

[7] S. Kobayashi and K. Nomizu, Foundations of Differential Geometry II, Interscience Publishers, 1969.

[8] H. Nakagawa and R. Takagi, On locally symmetric Kaehler submanifolds in a complex projective space, J. Math. Japan, 28 (1976), 638-667.

[9] K. Nomizu, Invariant affine connections on homogeneous spaces, Amer. J. Math., 76 (1954), 33-65.

[10] R. Takagi, Kählerian submanifolds in a complex projective space with second fundamental form of polynomial type, to appear.

[11] M. Takeuchi, Homogeneous Kähler submanifolds in complex projective spaces, Japan. J. Math., 4 (1978), 171-219.

[12] H. C. Wang, Closed manifolds with homogeneous complex structures, Amer. J. Math., 76 (1954), 1-32.

[13] J. A. Wolf, On the classification of hermitian symmetric spaces, J. Math. Mech., 13 (1964), 489-495.

Department of Matherratics

University of Tsukuba

Sakura-mura, Niihari-gun,

Ibaraki, Japan 